\title{
A NONLINEAR SECOND INITIAL BOUNDARY VALUE PROBLEM FOR THE HEAT EQUATION*
}

\author{
BY \\ C. Y. CHAN \\ Florida State University
}

1. Introduction. Mann and Wolf [6] proved the existence and uniqueness of an initial boundary value problem of a one-dimensional heat equation with zero initial temperature and nonlinear second boundary condition. Their result was improved by Roberts and Mann [9], and later on by Padmavally [8]. Using Schauder's fixed point theorem [10], Friedman [2] considered an $n$-dimensional linear parabolic differential equation with linear initial condition and nonlinear boundary condition involving the conormal.

We use a completely different approach to establish the existence and uniqueness of a solution for a nonlinear second initial boundary value problem consisting of a semilinear parabolic differential equation with linear initial and quasilinear boundary conditions. The arguments, similar to those of Duff [1] for the elliptic case, give the solution by successive approximations; in each step of the construction, we make use of the solution of the corresponding linear problem. The method can be used for the more general parabolic differential equation,

$$
\sum_{i, j=1}^{n} a_{i j}(x, t) \frac{\partial^{2} u}{\partial x_{i} \partial x_{i}}+\sum_{i=1}^{n} a_{i}(x, t) \frac{\partial u}{\partial x_{i}}+c(x, t) u-\frac{\partial u}{\partial t}=g(x, t ; u),
$$

since for this the strong maximum principle [7] holds, and the Neumann function exists $[3$, p. 155, 4, 5] under certain conditions on the coefficients and the domain of definition. For simplicity of discussion, we consider here an $n$-dimensional semilinear heat equation.

2. Statement of the problem. Let $D$ be a bounded convex n-dimensional domain in the real $n$-dimensional Euclidean space, $D^{-}$its closure and $\partial D$ its boundary. For every point $x=\left(x_{1}, x_{2}, \cdots x_{n}\right)$ of $\partial D$, there exists an $n$-dimensional neighborhood $V$ such that $V \cap \partial D$ can be represented for some $i(1 \leq i \leq n)$ in the form

$$
x_{i}=h\left(x_{1}, x_{2}, \cdots, x_{i-1}, x_{i+1}, \cdots, x_{n}\right)
$$

and the functions $h, D_{x} h, D_{x}^{2} h$ are Hölder continuous of exponent $\alpha$ where $0<\alpha<1$. Let $D \times(0, T]=\Omega, \partial D \times(0, T]=S$, and

$$
L=\sum_{i=1}^{n} \frac{\partial^{2}}{\partial x_{i}^{2}}-\frac{\partial}{\partial t}
$$

* Received April 2, 1970; revised version received July 26, 1970. This work is a portion of the author's doctoral dissertation at the University of Toronto, and was supported by a postgraduate scholarship from the Nationa! Research Council of Canada. 
Our problem is to find $u(x, t)$ satisfying the semilinear heat equation

$$
L u=g(x, t ; u) \text { in } \Omega
$$

under the initial condition

$$
u(x, 0)=\phi(x) \text { on } D^{-}
$$

and the quasilinear boundary condition

$$
\frac{\partial u(x, t)}{\partial n_{(x, t)}}+B(x, t ; u)=f(x, t) \text { on } S,
$$

where $g(x, t ; u), \phi(x), B(x, t ; u)$ and $f(x, t)$ are given functions, and $n_{(x, t)}$ is the outward normal to $S$ at the point $(x, t)$. We impose the following conditions:

(i) $g(x, t ; u)$ is twice continuously differentiable; $g_{u}(x, t ; u)$ is Hölder continuous when $(x, t) \in \Omega^{-}$and $u$ varies in a bounded set;

and

$$
0 \leq g_{u}(x, t ; u)<\infty
$$

$$
g(x, t ; 0)=0
$$

(ii) $\phi(x)$ is continuous in $D^{-}$;

(iii) $B(x, t ; u)$ is twice continuously differentiable when $(x, t) \in S^{-}$and $u$ varies in a bounded set; moreover

and

$$
B_{u}(x, t ; u)>0
$$

$$
B(x, t ; 0)=0 ;
$$

(iv) $f(x, t)$ is continuous on $S^{-}$.

For $n=3$, the problem can be interpreted physically as finding the temperature $u(x, t)$ of a convex, sufficiently smooth, homogeneous and isotropic body having an arbitrary initial distribution of temperature $\phi(x)$. Heat is generated in it at a rate proportional to $-g(x, t ; u)$, which is a nonincreasing function of $u$ (condition (2.4)) and satisfies (2.5). Heat transfer between the body at a higher temperature and its surroundings at a lower constant temperature $[6, \mathrm{pp} .163-164]$ is subject to a nonlinear condition (2.3). Thus $f(x, t)-B(x, t ; u)$ is a monotone decreasing function of $u$ (condition (2.6)) [6, pp. 163-164]. If $f(x, t) \equiv 0$ on $S^{-}$, then (2.7) implies that the temperature of the surroundings is zero [6, p. 164].

The main result of this work is the following theorem.

THEOREM. There exists a unique solution of the nonlinear second initial-boundary value problem (2.1)-(2.3).

In Sec. 3, we consider three auxiliary lemmas. The proof of the theorem is given in Sec. 4. If conditions (2.5) and (2.7) are replaced by $g(x, t ; m)=0$ and $B(x, t ; m)=0$ where $m$ is a constant, then (4.1) is replaced by

$$
u(x, 0 ; \lambda)-m=\lambda(\phi(x)-m) \text { on } D^{-} .
$$

Accordingly, we make the corresponding changes in the existence proof; for example, we start with

$$
u_{0}(x, t ; \lambda) \equiv u(x, t ; 0)=m
$$


in the successive approximations. In effect, the procedures of the proof remain the same.

3. Auxiliary lemmas. Let $L_{c}=L-c(x, t)$, where $c(x, t) \geq 0$ and $c(x, t)$ is Hölder continuous in $\Omega^{-}$. Also let

$$
B_{\tau}=(D \times[0, T]) \cap\{t=\tau\}
$$

$\Omega^{*}=D \times[0, T)$, and

$$
\psi_{\beta}=\frac{\partial}{\partial n_{(x, t)}}+\beta(x, t)
$$

where $\beta(x, t)$ is a continuous function on $S^{-}$. To define a Neumann function, we follow Friedman [3, p. 155].

Definition. A function $N(x, t ; \xi, \tau)$ defined and continuous for $(x, t ; \xi, \tau) \in \Omega^{-} \times \Omega^{*}$, $t>\tau$, is called a Neumann function of $L_{c} \imath=0$ in $\Omega$ if for any $0 \leq \tau<T$ and for any continuous function $\psi(x)$ on $B_{\tau}$ having a compact support, the function

$$
w(x, t)=\int_{B_{\tau}} N(x, t ; \xi, \tau) \psi(\xi) d \xi
$$

is a solution of $L_{c} v=0$ in $D \times(\tau, T]$ and satisfies

$$
\lim _{t \downarrow \tau} w(x, t)=\psi(x) \quad \text { for } x \in B_{\tau}^{-},
$$

and $\psi_{\beta} w(x, t)=0$ on $\partial D \times(\tau, T]$.

Let $N^{*}(x, t ; \xi, \tau)$ denote the Neumann function of the adjoint equation $L_{c}^{*} w=0$ in $\Omega^{*}$ corresponding to the boundary condition $\psi_{\beta} w=0$ on $\partial D \times[0, \tau)$. By Friedman [3, p. 155, pp. 82-84] and Itô [4], $N(x, t ; \xi, \tau)$ and $N^{*}(x, t ; \xi, \tau)$ exist and are unique, $L_{c} N(x, t ; \xi, \tau)=0$ for $(x, t) \in \Omega, L_{c}^{*} N^{*}(x, t ; \xi, \tau)=0$ for $(x, t) \in \Omega^{*}, \psi_{\beta} N(x, t ; \xi, \tau)=0$ for $(x, t) \in \partial D \times(\tau, T], \psi_{\beta} N^{*}(x, t ; \xi, \tau)=0$ for $(x, t) \in \partial D \times[0, \tau)$, and furthermore, $N(x, t ; \xi, \tau), N_{x}(x, t ; \xi, \tau), N_{x x}(x, t ; \xi, \tau)$ and $N_{\imath}(x, t ; \xi, \tau)$ are continuous functions of $(x, t ; \xi, \tau)$ in $\Omega \times \Omega^{*}, t>\tau$ while $N^{*}(x, t ; \xi, \tau), N_{x}^{*}(x, t ; \xi, \tau), N_{x x}^{*}(x, t ; \xi, \tau)$ and $N_{t}^{*}(x, t$; $\xi, \tau)$ are continuous functions of $(x, t ; \xi, \tau)$ in $\Omega^{*} \times \Omega, t<\tau$. The Neumann function can be constructed by the parametrix method used by Itô $[4,5]$.

Let $N(x, t ; \xi, \tau)$ be the Neumann function corresponding to the case when $c(x, t) \geq 0$ and $\beta(x, t) \geq 0$, and $N^{0}(x, t ; \xi, \tau)$ be that corresponding to the case when $c(x, t)$ and $\beta(x, t)$ are identically zero. Then,

Lemma 1. $N(x, t ; \xi, \tau) \leq N^{0}(x, t ; \xi, \tau)$.

Proof. In the Green's identity,

$$
v L_{c} u-u L_{c}^{*} v=\sum_{i=1}^{n} \frac{\partial}{\partial x_{i}}\left\{\sum_{i=1}^{n}\left(v \frac{\partial u}{\partial x_{i}}-u \frac{\partial v}{\partial x_{i}}\right)\right\}-\frac{\partial}{\partial t}(u v),
$$

let $u(y, \sigma)=N(y, \sigma ; \xi, \tau)$ and $v(y, \sigma)=N^{*}(y, \sigma ; x, t)$. Integrating this over the domain $D \times(\tau+\epsilon, t-\epsilon)$ and letting $\epsilon \rightarrow 0$, we have by the boundary condition

$$
N(x, i ; \xi, \tau)=N^{*}(\xi, \tau ; x, t)
$$

for any two points $(x, t)$ and $(\xi, \tau)$ in $\Omega$ with $t>\tau$. An argument similar to the proof of Theorem 11 of Friedman [3, pp. 44-45] gives for each $(\xi, \tau)$ in $\Omega^{*}$,

$$
N(x, t ; \xi, \tau)>0 \text { in } D \times(\tau, T] .
$$


From this and (3.1), it follows that

$$
N^{*}(x, t ; \xi, \tau)>0 \text { in } D \times[0, \tau)
$$

for each $(\xi, \tau)$ in $\Omega$.

Let $N_{\lambda}(x, t ; \xi, \tau)$ be the Neumann function of $L_{c} w=0$ corresponding to the boundary condition $\psi_{\lambda} N_{\lambda}(x, t ; \xi, \tau)=0$, where $\lambda(x, t) \geq 0$. Then the Green's identity gives

$$
\begin{array}{r}
N_{\lambda}(x, t ; \xi, \tau)-N(x, t ; \xi, \tau)=-\int_{\tau}^{t} \int_{\partial D} N_{\lambda}^{*}(y, \sigma ; x, t) N(y, \sigma, \xi, \tau) \\
\cdot\{\lambda(y, \sigma)-\beta(y, \sigma)\} d A_{\nu} d \sigma,
\end{array}
$$

which gives

$$
\delta N(x, t ; \xi, \tau)=-\int_{\tau}^{t} \int_{\partial D} N^{*}(y, \sigma ; x, t) N(y, \sigma ; \xi, \tau) \delta \beta(y, \sigma) d A_{\nu} d \sigma .
$$

Similarly, let $N_{b}(x, t ; \xi, \tau)$ be the Neumann function of $L_{b} w=0$ corresponding to $\psi_{\beta} w=0$ with $b(x, t) \geq 0$. Then

$$
\begin{aligned}
& N_{b}(x, t ; \xi, \tau)-N(x, t ; \xi, \tau)=-\int_{\tau}^{t} \int_{D} N_{b}^{*}(y, \sigma ; x, t) N(y, \sigma ; \xi, \tau) \\
& \cdot\{b(y, \sigma)-c(y, \sigma)\} d V_{\nu} d \sigma,
\end{aligned}
$$

which gives

$$
\delta N(x, t ; \xi, \tau)=-\int_{\tau}^{t} \int_{D} N^{*}(y, \sigma ; x, t) N(y, \sigma ; \xi, \tau) \delta c(y, \sigma) d V_{\nu} d \sigma .
$$

Thus from (3.2), (3.3), (3.5) and (3.7), $N(x, t ; \xi, \tau) \leq N^{0}(x, t ; \xi, \tau)$ follows.

In what follows, let $k_{1}, k_{2}, k_{3}, \cdots, k_{11}$ denote appropriate positive constants. For convenience of reference, we state the following lemma, whose proof can be found in Friedman [3, p. 146].

LEMma 2. If $w$ is a solution of $L_{c} w=0$ in $\Omega, \psi_{\beta} w=f(x, t)$ on $S$ and $w(x, 0)=\phi(x)$ on $D^{-}$, then for all $(x, t) \in \Omega$,

$$
\left.|w(x, t)| \leq k_{1} \text { (1.u.b. }|f|+\underset{s^{-}}{\text {l.u.b. }}|\phi|\right),
$$

where $k_{1}$ is a constant depending only on $L_{c}, \beta$ and $\Omega^{-}$.

LeMMa 3. Let

$$
\begin{aligned}
\theta^{*}(\xi, \tau ; x, t)= & k_{2} \int_{\tau}^{t} \int_{D} N^{0}(y, \sigma ; \xi, \tau) N^{0^{*}}(y, \sigma ; x, t) d V_{\nu} d \sigma \\
& +k_{3} \int_{\tau}^{t} \int_{\partial D} N^{0}(y, \sigma ; \xi, \tau) N^{0^{*}}(y, \sigma ; x, t) d A_{\nu} d \sigma .
\end{aligned}
$$

Then

$$
\int_{D} \theta^{*}(\xi, 0, x, t) d V_{\xi}+\int_{0}^{t} \int_{\partial D} \theta^{*}(\xi, \tau ; x, t) d A_{\xi} d \tau \leq k_{4}
$$

where $k_{4}$ is independent of $(x, t)$. 
Proof. Let $L^{*}$ be the adjoint of $L$. It follows from the Green's identity that $\theta^{*}(\xi, \tau ; x, t)$ is the solution of

$$
\begin{aligned}
L^{*} \theta^{*}(\xi, \tau ; x, t) & =-k_{2} N^{0^{*}}(\xi, \tau ; x, t) \quad \text { in } D \times[0, t), \\
\theta^{*}(\xi, t ; x, t) & =0 \quad \text { on } \Omega^{-} \cap\{t=t\},
\end{aligned}
$$

and

$$
\frac{\partial \theta^{*}(\xi, \tau ; x, t)}{\partial n_{(\xi, \tau)}}=k_{3} N^{0^{*}}(\xi, \tau ; x, t) \quad \text { on } \partial D \times[0, t) .
$$

Let $w(x, t)$ be the solution of $L w=0$ in $\Omega, w(x, 0)=1$ on $D^{-}$, and $\partial w(x, t) / \partial n_{(x, t)}=1$ on $S$. From Lemma $2,|w(x, t)| \leq k_{5}$, a constant.

In the Green's identity, let $v=\theta^{*}(y, \sigma ; x, t)$ and $u=w(y, \sigma)$. Integrating this over the domain $D \times(\epsilon, t-\epsilon)$, and letting $\epsilon \rightarrow 0$, we have

$$
\begin{aligned}
\int_{D} \theta^{*}(\xi, 0 ; x, t) d V_{\xi}+\int_{0}^{t} \int_{\partial D} \theta^{*}(\xi, \tau ; x, t) d A_{\xi} d \tau \\
\quad=k_{2} \int_{0}^{t} \int_{D} w(\xi, \tau) N^{0^{*}}(\xi, \tau ; x, t) d V_{\xi} d \tau+k_{3} \int_{0}^{t} \int_{\partial D} w(\xi, \tau) N^{0^{*}}(\xi, \tau ; x, t) d A_{\xi} d \tau .
\end{aligned}
$$

Hence

$$
\begin{aligned}
\int_{D} \theta^{*}(\xi, 0 ; x, t) & d V_{\xi}+\int_{0}^{t} \int_{\partial D} \theta^{*}(\xi, \tau ; x, t) d A_{\xi} d \tau \\
& \leq k_{2} k_{5} \int_{0}^{t} \int_{D} N^{0^{*}}(\xi, \tau ; x, t) d V_{\xi} d \tau+k_{3} k_{5} \int_{0}^{t} \int_{\partial D} N^{0^{*}}(\xi, \tau ; x, t) d A_{\xi} d \tau .
\end{aligned}
$$

The right-hand side of the inequality is the solution of $L z=-k_{2} k_{5}$ in $\Omega, z(x, 0)=0$ on $D^{-}$and $\partial z(x, t) / \partial n_{(x, t)}=k_{3} k_{5}$ on $S$. Hence from Lemma 2

$$
|z(x, t)| \leq k_{6} k_{5}\left(k_{2}+k_{3}\right) \text {. }
$$

Thus the lemma is proved.

4. Proof of the theorem. Uniqueness: Suppose $u_{1}(x, t)$ and $u_{2}(x, t)$ are two distinct solutions of our problem. Without loss of generality, let us assume that $u_{2}(x, t)>$ $u_{1}(x, t)$ at some point of $\Omega$. Then the function, $u(x, t)=u_{2}(x, t)-u_{1}(x, t)$ satisfies

$$
L u-g_{u}\left(x, t ; u_{3}\right) u=0 \text { in } \Omega \text {, }
$$

where $u_{3}$ lies between $u_{1}$ and $u_{2}$. Since $u(x, 0)=0$ on $D^{-}$, we have by the weak maximum principle [7] that it attains its maximum at some point, say $\left(x_{0}, t_{0}\right)$, of $S$. Hence $\partial u\left(x_{0}, t_{0}\right) / \partial n_{\left(x_{0}, t_{0}\right)} \geq 0$, but

$$
\frac{\partial u\left(x_{0}, t_{0}\right)}{\partial n_{\left(x_{0}, t_{0}\right)}}=B\left(x_{0}, t_{0} ; u_{1}\right)-B\left(x_{0}, t_{0} ; u_{2}\right)<0
$$

by (2.6). Therefore, the solution is unique.

Existence: Let $\lambda$ be a parameter such that $0 \leq \lambda \leq 1$. If $u(x, t ; \lambda)$ is the solution of

$$
\begin{gathered}
L u(x, t ; \lambda)=g(x, t ; u(x, t ; \lambda)) \quad \text { in } \Omega, \\
\frac{\partial u(x, t ; \lambda)}{\partial n_{(x, t)}}+B(x, t ; u(x, t ; \lambda))=\lambda f(x, t) \quad \text { on } S
\end{gathered}
$$


and

$$
u(x, 0 ; \lambda)=\lambda \phi(x) \text { on } D^{-},
$$

then $v(x, t ; \lambda) \equiv \partial u(x, t ; \lambda) / \partial \lambda$ satisfies

and

$$
\begin{aligned}
& L_{\partial_{x}} v(x, t ; \lambda)=0 \text { in } \Omega, \\
& \psi_{B_{u}} v(x, t ; \lambda)=f(x, t) \text { on } S
\end{aligned}
$$

$$
v(x, 0 ; \lambda)=\phi(x) \text { on } D^{-} .
$$

Now if $u(x, t ; \lambda)$ is already known, then by the Green's identity

$$
v(x, t ; \lambda)=\int_{D} N(x, t ; \xi, 0 ; \lambda) \phi(\xi) d V_{\xi}+\int_{0}^{t} \int_{\partial D} N(x, t ; \xi, \tau ; \lambda) f(\xi, \tau) d A_{\xi} d \tau,
$$

where $N(x, t ; \xi, \tau ; \lambda)$ is the Neumann function of (4.2) corresponding to the boundary condition $\psi_{B_{u}} v(x, t ; \lambda)=0$ on $S$. But as $\lambda$ varies, $u(x, t ; \lambda)$ changes, and this in turn affects the Neumann function. By (3.5) and (3.7), we have

$$
\begin{gathered}
\delta N(x, t ; \xi, \tau ; \lambda)=-\int_{\tau}^{t} \int_{D} N^{*}(y, \sigma ; x, t ; \lambda) N(y, \sigma ; \xi, \tau ; \lambda) \delta g_{u}(y, \sigma, u(y, \sigma ; \lambda)) d V_{\nu} d \sigma \\
-\int_{\tau}^{t} \int_{\partial D} N^{*}(y, \sigma ; x, t ; \lambda) N(y, \sigma ; \xi, \tau ; \lambda) \delta B_{u}(y, \sigma ; u(y, \sigma ; \lambda)) d A_{\nu} d \sigma .
\end{gathered}
$$

Thus to determine $u(x, t ; \lambda)$ and $N(x, t ; \xi, \tau ; \lambda)$, we have the following system of integrodifferential equations:

$$
\frac{\partial u(x, t ; \lambda)}{\partial \lambda}=\int_{D} N(x, t ; \xi, 0 ; \lambda) \phi(\xi) d V_{\xi}+\int_{0}^{t} \int_{\partial D} N(x, t ; \xi, \tau ; \lambda) f(\xi, \tau) d A_{\xi} d \tau
$$

and

$$
\begin{gathered}
\frac{\partial N(x, t ; \xi, \tau ; \lambda)}{\partial \lambda}=-\int_{\tau}^{t} \int_{D} N^{*}(y, \sigma ; x, t ; \lambda) N(y, \sigma ; \xi, \tau ; \lambda) \frac{\partial g_{u}(y, \sigma ; u(y, \sigma ; \lambda))}{\partial \lambda} d V_{\nu} d \sigma \\
-\int_{\tau}^{t} \int_{\partial D} N^{*}(y, \sigma ; x, t ; \lambda) N(y, \sigma ; \xi, \tau ; \lambda) \frac{\partial B_{u}(y, \sigma ; u(y, \sigma ; \lambda))}{\partial \lambda} d A_{\nu} d \sigma
\end{gathered}
$$

with $u(x, t ; 0) \equiv 0$.

By Lemma 2,

\section{Hence}

$$
\left.|v(x, t ; \lambda)| \leq \underset{\mathcal{S}^{-}}{k_{7}(1 . u . b .}|f|+\underset{D^{-}}{\operatorname{l.u.b}}|\phi|\right) .
$$

$$
\left.u(x, t ; \lambda) \leq k_{7} \text { (1.u.b. }|f|+\underset{s^{-}}{\text {l.u.b. }}|\phi|\right)
$$

since $0 \leq \lambda \leq 1$. We now prove the existence in the theorem by successive approximations.

Let $u_{0}(x, t ; \lambda) \equiv u(x, t ; 0)=0$. For $n=1,2,3, \cdots$, let $u_{n}(x, t ; 0) \equiv 0$, and $\frac{\partial u_{n}(x, t ; \lambda)}{\partial \lambda}=\int_{D} N_{n-1}(x, t ; \xi, 0 ; \lambda) \phi(\xi) d V_{\xi}+\int_{0}^{t} \int_{\partial D} N_{n-1}(x, t ; \xi, \tau ; \lambda) f(\xi, \tau) d A_{\xi} d \tau$ 
where $N_{n}(x, t ; \xi, \tau ; \lambda)$ is the Neumann function of the differential equation

$$
L v(x, t ; \lambda)=g_{u}\left(x, t ; u_{n}(x, t ; \lambda)\right) v(x, t ; \lambda)
$$

corresponding to the boundary condition

$$
\frac{\partial v(x, t ; \lambda)}{\partial n_{(x, t)}}+B_{u}\left(x, t ; u_{n}(x, t ; \lambda)\right) v(x, t ; \lambda)=0 .
$$

Thus we can find $N_{0}(x, t ; \xi, \tau ; \lambda), u_{1}(x, t ; \lambda), N_{1}(x, t ; \xi, \tau ; \lambda)$, and so on successively.

Since $g(x, t ; u)$ and $B(x, t ; u)$ are twice continuously differentiable, we have by (4.6) that $g_{u u}$ and $B_{u u}$ are bounded. Let $\left|g_{u u}\right| \leq k_{2}$ and $\left|B_{u u}\right| \leq k_{3}$. Also let

$$
\rho_{n}(\lambda)=\max _{(x, t) \in \Omega}\left|u_{n}(x, t ; \lambda)-u_{n-1}(x, t ; \lambda)\right| \text {. }
$$

Then

$$
\left|g_{u}\left(x, t ; u_{n}(x, t ; \lambda)\right)-g_{u}\left(x, t ; u_{n-1}(x, t ; \lambda)\right)\right| \leq k_{2} \rho_{n}(\lambda)
$$

and

$$
\left|B_{u}\left(x, t ; u_{n}(x, t ; \lambda)\right)-B_{u}\left(x, t ; u_{n-1}(x, t ; \lambda)\right)\right| \leq k_{3} \rho_{n}(\lambda) .
$$

These together with (3.4), (3.6), Lemma 1 and the definition of $\theta^{*}(\xi, \tau ; x, t)$ in Lemma 3 give

$$
\left|N_{n}(x, t ; \xi, \tau ; \lambda)-N_{n-1}(x, t ; \xi, \tau ; \lambda)\right| \leq \rho_{n}(\lambda) \theta^{*}(\xi, \tau ; x, t) .
$$

Let $|\phi(x)| \leq k_{8},|f(x, t)| \leq k_{9}$ and $k_{10}=\max \left\{k_{8}, k_{9}\right\}$. Then from (4.7) and (4.9), we have

$$
\begin{aligned}
& \left|\frac{\partial u_{n+1}(x, t ; \lambda)}{\partial \lambda}-\frac{\partial u_{n}(x, t ; \lambda)}{\partial \lambda}\right| \\
& \leq k_{10} \rho_{n}(\lambda)\left\{\int_{D} \theta^{*}(\xi, 0 ; x, t) d V_{\xi}+\int_{0}^{t} \int_{\partial D} \theta^{*}(\xi, \tau ; x, t) d A_{\xi} d \tau\right\} \leq k_{10} \rho_{n}(\lambda) k_{4}
\end{aligned}
$$

by Lemma 3. Since $u_{n}(x, t ; 0)=0$, we have from (4.10)

$$
\left|u_{n+1}(x, t ; \lambda)-u_{n}(x, t ; \lambda)\right| \leq k_{4} k_{10} \int_{0}^{\lambda} \rho_{n}(r) d r,
$$

which is independent of $(x, t)$. By (4.8)

$$
\rho_{n+1}(\lambda) \leq k_{4} k_{10} \int_{0}^{\lambda} \rho_{n}(r) d r
$$

Since $u_{0}(x, t ; \lambda)=0$, we have

$$
\rho_{1}(\lambda)=\max _{(x, t) \in \Omega^{-}}\left|u_{1}(x, t ; \lambda)\right| .
$$

By (4.6), $\rho_{1}(\lambda) \leq k_{11}$. It follows from induction that

$$
\rho_{n}(\lambda) \leq \frac{k_{11}\left(k_{4} k_{10} \lambda\right)^{n-1}}{(n-1) !} .
$$

Therefore, $\sum_{n=0}^{\infty}\left[u_{n+1}(x, t ; \lambda)-u_{n}(x, t ; \lambda)\right]$ converges absolutely and uniformly in $(x, t)$. Let $u(x, t ; \lambda)$ be the limit. Except at the point of singularity $(x, t)=(\xi, \tau)$ of $N^{0}(x, t ; \xi, \tau)$, 
it follows from (4.9) that the sequence $\left\{N_{n}(x, t ; \xi, \tau ; \lambda)\right\}$ converges uniformly to a limit, say $N(x, t ; \xi, \tau ; \lambda)$. Thus for $(x, t) \neq(\xi, \tau), N(x, t ; \xi, \tau ; \lambda)$ is continuous and furthermore, from (4.3), it depends continuously on the coefficient of the partial differential equation and on the boundary condition. Therefore $N(x, t ; \xi, \tau ; \lambda)$ is the Neumann function of (4.2) corresponding to $\psi_{B_{u}} v(x, t ; \lambda)=0$ on $S$. Hence from $(4.3) \partial N(x, t ; \xi, \tau ; \lambda) / \partial \lambda$ is given by (4.5). Since $u_{0}(x, t ; \lambda)=0$, we have from (4.10) and (4.11) that $\partial u_{n}(x, t ; \lambda) / \partial \lambda$ converges uniformly and absolutely. As $n \rightarrow \infty$, (4.7) becomes (4.4). Thus $u(x, t ; \lambda)$ and $N(x, t ; \xi, \tau ; \lambda)$ satisfy the integro-differential equations (4.4) and (4.5) with $u(x, t ; 0) \equiv 0$. Hence $u(x, t ; 1)$ is the solution to our problem.

\section{REFERENCES}

[1] G. F. D. Duff, A quasi-linear boundary value problem, Trans. Roy. Soc. Canada Sect. III. (3) 49, 7-17 (1955)

[2] A. Friedman, Generalized heat transfer between solids and gases under nonlinear boundary conditions, J. Math. Mech. 8, 161-183 (1959)

[3] A. Friedman, Partial differential equations of parabolic type, Prentice-Hall, Englewood Cliffs, N. J., 1964, pp. 44-45, 82-84, 146, 155

[4] S. Itô, $A$ boundary value problem of partial differential equations of parabolic type, Duke Math. J. 24, 299-312 (1957)

[5] S. Itô, A remark on my paper " $A$ boundary value problem of partial differential equations of parabolic type" in Duke Mathematical Journal, Proc. Japan Acad. 34, 463-465 (1958)

[6] W. R. Mann and F. Wolf, Heat transfer between solids and gases under nonlinear boundary conditions, Quart. Appl. Math. 9, 163-184 (1951)

[7] L. Nirenberg, A strong maximum principle for parabolic equations, Comm. Pure Appl. Math. 6, 167-177 (1953)

[8] K. Padmavally, On a non-linear integral equation, J. Math. Mech. 7, 533-555 (1958)

[9] J. H. Roberts and W. R. Mann, On a certain nonlinear integral equation of the Volterra type, Pacific J. Math. 1, 431-445 (1951)

[10] J. Schauder, Der Fixpunktsatz in Funktionalräumen, Studia Math. 2, 171-180 (1930) 